\title{
1p/19q Codeletion Negative
}

National Cancer Institute

\section{Source}

National Cancer Institute. 1p/19q Codeletion Negative. NCI Thesaurus. Code C153099.

A finding indicating that concurrent deletion of chromosome arms $1 p$ and $19 q$ is not detected in a sample. 